\title{
Migración, género y trabajo sexual: una perspectiva compleja
}

\section{Migration, Gender and Sex Work: A Complex Perspective}

\section{RESUMEN}

El trabajo sexual es una realidad objeto de un importante debate en el contexto de las ideas y la praxis feministas, pero casi siempre es planteado desde enfoques problemáticos, ya sea por reduccionismos de distinta clase, falacias argumentativas o saltos conceptuales. Uno de los objetivos de este texto es identificar algunos de estos problemas. Sin embargo, el objeto clave aquí es el de organizar un boceto que permita ofrecer una mirada complejizadora de un tema no menos sencillo. Así, este estudio breve recopila algunas de las reflexiones fundamentales a la hora de plantear la prostitución dentro del espacio global capitalista y como institución de carácter patriarcal. A través de este tema se conectan los fenómenos de la globalización y del territorio, que muestran una resignificación dialéctica. El artículo también aborda brevemente el caso de la ciudad de Murcia, como ejemplo de análisis aplicado a una política concreta.

Palabras clave: trabajo sexual, prostitución, género, migración, territorio.

\begin{abstract}
Sex work is the center of an important debate in the context of feminist ideas and actions, but at the same time it is almost always thought from a problematic perspective, whether it be reductionisms of any king, argumentative falacies or conceptual gaps. One of the objects of this very text is to identify some of those problems. Nevertheless, the main purpose of this work is to draw a sketch which may offer an insight of the complexity of the matter. In doing so, this brief work gathers some of the most important reflexions about prostitution within the capitalist globe and as a patriarchal institution. It is through this theme that globalization and territory happen to be connected as phenomena, and be dialectically reificated. This text also breifly comments the situation of Murcia as an example of applied analysis.
\end{abstract}

Keywords: sex work, prostitution, gender, migration, territory.

\section{SUMARIO}

0, Introducción; 1, Dinámicas estructurales: 1.1. Globalización, transformación y redes transnacionales de migración, 1.2. Feminización del trabajo y trabajo de cuidados, 1.3. Migraicón y trabajo sexual; 2, Trabajo sexual: 2.1. Mitificaciones feministas, malentendidos sociológicos, 2.2. Trabajo, trabajo sexual y cuerpo, 2.3., Murcia: las consecuencias empíricas de la prohibición; 3, Conclusiones: para pensar el trabajo sexual -Bibliografía y documentación. 


\section{INTRODUCCIÓN.}

La prostitución es un tema complejo y con muchos pliegues, en torno al cual se organiza un debate polarizado y rara vez matizado. Además de las posturas que se organizan en torno a la prohibición y a la regularizacion acrítica, hay que comentar que el estudio de la prostitución se plantea normalmente en torno al cuerpo de las propias mujeres prostitutas, y a menudo se aproxima a las dinámicas patriarcales implícitas en este trabajo. También se debate el hecho de que sea o no «trabajo» (o dicho más técnicamente, «empleo»), si bien en este texto vamos a tomar la perspectiva de la Global Network of Sex Work Projects (2015), que asume que es trabajo en la medida en que existe un intercambio económico directo. Para analizar este tema adecuadamente hay que separar este fenómeno de otros, como el tráfico con fines sexuales o la explotación sexual en general. También hay que añadir que existen otras formas de trabajo sexual; es precisamente esta heterogeneidad (y la diversidad de los medios implicados) la que se trata de categorizarse bajo el «trabajo sexual» como término paraguas, y bajo el concepto de «industria sexual» para hacer hincapié en la dinámica estructural. Sobre el «trabajo sexual» en este sentido haremos una reconstrucción conceptual a través del concepto de «trabajo» en general.

Este estudio breve recopila algunas de las reflexiones fundamentales a la hora de plantear la prostitución dentro del espacio global capitalista y como institución de carácter patriarcal, e incide en el caso particular de Murcia. A través de este tema se conectan los fenómenos de la globalización y del territorio, que muestran una resignificación dialéctica. En la primera parte se presentan algunos de los hilos que conectan la globalización, el territorio y el género, bajo el rótulo de dinámicas estructurales; la segunda parte indaga de forma breve en la realidad de la prostitución a nivel de trabajo, raza y género; y la tercera parte muestra el caso de Murcia a través de la Ordenanza para luchar contra la prostitución en el Municipio de Murcia.

\section{DINÁMICAS ESTRUCTURALES.}

\section{1. Globalización, transformación y redes transnacionales de migración}

Aunque la globalización no es un fenómeno nuevo -desde luego no en el sentido de que haya habido migraciones masivas, o grandes movimientos culturales-, adquiere un nuevo sentido a partir de la segunda mitad del siglo XX. No está claro que exista un rasgo definitorio de este fenómeno, por lo que se suele acudir a una comprensión multi-factorial e histórica. Desde este planteamiento previo, la globalización dependería de la mundialización de varios fenómenos históricamente específicos: (a) el flujo transnacional de capitales, facilitado enormemente por (b) las tecnologías digitales y (c) el desarrollo y la privatización bancaria, (d) la unidad de los Estados-nación (como administradores de la producción en un territorio determinado) en los dogmas del programa político neoliberal, y todo esto condicionado por (e) el desarrollo de los medios de transporte y de (f) las 
redes poblacionales, que funcionan como unidades básicas de la producción. Las bases internacionales fueron puestas mediante dos grandes intervenciones: la conferencia de Yalta -en la que Roosevelt sugería crear una Organización de las Naciones Unidas para mantener la paz entre naciones- y los Acuerdos de Bretton Woods -en los que se aprobó la construcción de una serie de instituciones y tratados económicos para fomentar el libre comercio. Así se firmó el nuevo orden económico-institucional mundial que sentaría las bases de la globalización, bajo el reinado ideológico del Estado social liberal.

Por motivos de espacio, vamos a obviar las distintas condiciones y razones que conducen a los Estados de mayor peso en la economía mundial a pasar de las políticas intervencionistas (popularmente asociadas a la figura y las ideas liberales de Keynes) a políticas «neoliberales», políticas de privatización y financiarización del entorno económico que conducen de nuevo a la acumulación de las rentas y la pauperización de la clase trabajadora en general (Piketty, 2014). Aquí se enmarcan distintos procesos -económicos, legales, geográficos-, que forman en su unidad un híbrido complejo. Haremos sólo dos brevísimas referencias: la lectura actual que el filósofo Giorgio Agamben (2016) hace de El capitalismo como religión, texto clave de W. Benjamin, con el mismo nombre; y el excelente libro de López y Rodríguez (2010).

El proceso (quizá sería más preciso usar el plural aquí) de globalización ha determinado la transformación de los enclaves poblacionales, en términos económicos como espaciales. La tensión entre ciudad y campo se ha reconvertido, haciendo dela ciudad sede de los procesos propios del neoliberalismo, esencialmente la privatización y venta de los espacios, destinados a actividades de alto valor agregado (Theodore et al., 2009). Esto supone el desplazamiento general y la «reorganización espacial de la actividad económica» por medio del valor general de producción y la conexión con el entramado global (Sassen, 2003:38-42). En resumen, en el contexto de los actuales procesos de globalización de las ciudades, «el ascenso de sectores digitalizados... y desmaterializados, como el financiero, ha diluido ese tipo de articulación de la economía nacional..., y ha creado subeconomías orientadas al mercado mundial» (íb.:42). Sassen defiende que, en respuesta a estas «geografías o paisajes de la globalización» surgen «contrageografías de la globalización», cuya delimitación está construida -como es el caso de las redes sociales-necesariamente por las estructuras que conforman los primeros paisajes.

En este contexto de redefinición de los espacios globales y locales aparecen, por primera vez de forma sostenida e institucionalizada en la historia, cadenas globales de migraciones. Aparecen tanto en el «sector formal» como en el «informal», o sea, estén o no contempladas en el entramado burocrático (Agustín, 2006; Scott, 2019). Estas cadenas que, en su conjunto, forman redes, se ven estructuradas por factores políticos -en la medida en que el marco legal hace referencia directa al entramado político-burocrático-, económicos -ya que los procesos económicos se complejizan y atrapan con sus dinámicas a grupos cada vez más numerosos- y sociológicos pues remiten a las formas en las que se organizan las relaciones sociales- a lo largo de la historia de las últimas décadas. 
El dominio histórico del Norte global sobre el Sur, tanto en social como en lo económico (Dussel, 2013:185-202), ha determinado estructuralmente las migraciones contemporáneas, tanto internas como externas. Por sus relaciones históricas con América Latina y el resto de Europa, España es un buen ejemplo del nudo que produce y organiza los desplazamientos masivos de las últimas décadas, en lo que Sassen (2015:60-61) plantea como parte visible del nuevo régimen de expulsiones propio de la nueva economía global. Pero hay que hacer una distinción: «las tasas de desempleo, emigración, desalojos, pobreza y suicidios son variables útiles en los países del Norte global; para el Sur global, posiblemente sean más útiles las tasas de desplazamiento debido a guerras, enfermedades y hambrunas» (ib.:69-75).

Hay que incidir, además, en que la mayor parte de migraciones se producen entre países del Sur: «el 80 por ciento de los refugiados del mundo están en países del Sur global» (íb.). En los países del Norte, donde las actividades de alto valor agregado son el principal atractivo de los empresarios, la migración tiende a presentarse por las patronales como un mecanismo más para la devaluación de los salarios, organizándose en torno a la población migrante -y a costa suya- «etnoestratificación laboral, donde los nichos de trabajo ocupados por los inmigrantes requieren baja cualificación laboral y prácticamente no producen una movilidad ascendente» (Valero-Matas et al., 2014). En cambio, los países del Sur, en relación al Norte global, tienden a exportar trabajadores, lo que se traduce como un «envío de remesas que en muchos países representa una fuente importante de ingresos de moneda extranjera para los gobiernos», y en «instrumentos... para amortiguar el desempleo y la deuda externa» (Sassen, 2003:73 y ss.). El contraste resulta enorme: para 2007, alrededor del $45 \%$ de la población migrante residente en España enviaba remesas habitualmente o "de forma ocasional», siendo especialmente notable el caso de originarios de países andinos y africanos y de la Europa «no desarrollada» (INE, 2008:127-129).

Por razones de espacio, en lo sucesivo nos referiremos a las cadenas de migración que se dan del Sur al Norte global, para mantener como ejemplo el caso español.

\section{2. Feminización del trabajo y trabajo de cuidados}

Las cadenas globales de migración Sur-Norte se definen en buena medida a través del género y por medio de la familia como institución; las relaciones de género explican «quiénes y por qué razones emigran, cómo se toma la decisión de hacerlo, así como las consecuencias sobre las propias personas migrantes y los impactos tanto en las comunidades de origen como de destino» (Molpeceres, 2012). Por esto, de la misma forma que se da una etnoestratificación en la población migrante, el género es un factor fundamental para considerar cómo se dan y se transforman las relaciones dentro de estas comunidades en un mismo espacio, así como a lo largo de las cadenas transnacionales. Interesa también la distinción entre mujeres migrantes y autóctonas, en la medida en que esta diferencia organiza la etnoestratificación. 
La mayor participación de las mujeres españolas en el trabajo a partir del último cuarto del siglo XX supuso, junto con las medidas anticonceptivas y de conciliación, un paso clave a la hora de que las propias mujeres adquirieran visibilidad y, ante todo, autonomía personal e independencia; sin embargo, la división sexual del trabajo pasó prácticamente desapercibida, traduciéndose en este trasvase familiatrabajo en una importante segregación ocupacional. En este contexto, las mujeres están infrarrepresentadas en trabajos de extracción y transformación de recursos (especialmente en la industria manufacturera, en construcción, agricultura, pesca y ganadería y transporte), mientras que están sobrerrepresentadas en los trabajos de cuidado y de servicios en general (en actividades sanitarias y de servicios sociales, trabajo doméstico y administración) (Millán et al., 2015). Aunque no tiende a destacarse esta división sexual del trabajo, es parte connatural del más reconocido «techo de cristal», que incide en la diferencia de sueldos, y que afecta enormemente a la organización general de las cadenas de migración.

La bibliografía (ver Oso y Parella, 2012; Molpeceres, 2012; Barañano y Marchetti, 2016) incide en la nueva «internacionalización del trabajo reproductivo»y en la «crisis de los cui-dados» que se organiza globalmente por medio de las «cadenas globales de cuidado» (término popularizado por Hochschild, 2001; ver igualmente Orozco, 2007), así como en el «aumento creciente de la demanda de cuidados, especialmente... en relación, cada vez más, con personas mayores y dependientes, como ha sucedido en... Europa, y muy marcadamente en Europa del Sur» (Barañano y Marchetti, 2016). En pocas palabras: a raíz de la incorporación al trabajo de las mujeres nacionales -y por lo tanto del «doble sueldo familiar»-, de la «flexibilización» del trabajo y del tiempo de trabajo y del aumento de las cargas de los cuidados (que se puede observar en el desarrollo del sistema de atención a la dependencia en España, por ejemplo; ver Camacho et al., 2008), catalizado por la «ausencia de una cultura pública del cuidado» y por «un Estado del bienestar insuficientemente desarrollado»(Oso y Parella, op.cit.), las mujeres migrantes forman una «cadena global de cuidados».

No disponemos de datos de ocupación laboral desagregados por sexo; por otra parte, la Encuesta Nacional de Inmigrantes data de 2007, y todo recuento posterior tiene en cuenta sola y exclusivamente a los migrantes en situación regular, con lo que no contamos con evidencias suficientes para inferir conclusiones exactas a una escala nacional. Sí disponemos de trabajos en torno a las dinámicas laborales en la población migrante, sin embargo, y esencialmente en la demanda por sectores ocupacionales (ver Oso y Parela, op.cit.). Hay que destacar el trabajo de Molpeceres (2012), que ofrece una información y un análisis necesarios e ilustrativos, en base a diferentes fuentes estadísticas.

De acuerdo con el desarrollo de esta autora, las mujeres latinoamericanas (procedentes en especial de Ecuador, Colombia y Bolivia, y en menor medida de Argentina) tienen un nivel de actividad alto y sus ocupaciones son principalmente la hostelería y el servicio doméstico; por otra parte, su nivel educativo es de secundaria y, en el caso de las mujeres argentinas, entre la educación secundaria y la superior. Son mujeres en la treintena que migran para encontrar un empleo 
y reagruparse con su familia, siendo normalmente sus parejas -hombres padres de la familia- quienes emigran antes y encabezan el traslado familiar. Aunque la autora clasifica en cuatro perfiles distintos a las mujeres de estas nacionalidades, la migración andina a España es en general de un perfil muy similar, y la única diferencia notable en términos laborales es la movilidad social de las mujeres argentinas, debido seguramente al nivel educativo superior.

Otros dos perfiles que convergen en muchos puntos son los de las mujeres alemanas y las mujeres británicas, que migran en buena medida por jubilación (la media varía de los 45 a los 51), pero también por estudios y por trabajo; su nivel educativo es alto, y su nivel de actividad laboral es mucho menor, destacando entre sus profesiones los servicios empresariales, las ac-tividades inmobiliarias, la educación y la comunicación. Son mujeres con mayores facilidades económicas y mayor capital social.

Las mujeres chinas, rumanas y marroquíes forman tres perfiles con grandes divergencias, finalmente. En el primer y segundo caso son mujeres con estudios secundarios y superiores, y con una gran actividad laboral; las mujeres chinas se dedican fundamentalmente a hostelería y comercio, mientras que las mujeres rumanas muestran un alto nivel de autoempleo, y también se dedican al trabajo doméstico. Las mujeres marroquíes, por último, muestran unos niveles de estudios y de actividad bajos, haciéndose cargo esencialmente de las cargas familiares y no siendo parte del núcleo laboral familiar. Por último, son jóvenes de mediana edad.

El gran determinante a partir del cual se construyen todos estos perfiles es el origen, que estructura a su alrededor el resto de variables relevantes en el contexto socioeconómico. Se ha visto que una parte fundamental en la construcción de los perfiles de mujeres migrantes es el trabajo de cuidados y de servicios en general, como ya habíamos adelantado. También hemos destacado la falta de datos precisos y de reconstrucciones fieles, al margen de un solo estudio (Molpeceres, 2012). Esta situación se va a repetir y complejizar al tratar la prostitución, un trabajo profundamente estigmatizado y en torno al cual parecen levantarse toda una serie de resistencias que enconan el debate y contaminan el estudio.

\subsection{Migración y trabajo sexual}

Aunque las relaciones entre la migración y el trabajo sexual no son difíciles de establecer, sí es complicado situarlas con claridad. La información disponible proviene fundamentalmente de la organización de las propias trabajadoras y de sus testimonios en estudios cualitativos.

Seguramente la fuente a gran escala más fiable sea TAMPEP (The European Network for the Promotion of Rights and Health among Migrant Sex Workers), que ha colaborado con la Unión Europea puntualmente. Sin embargo, su último informe a escala europea («Sex Work in Europe: A Mapping of the Prostitution Scene in 25 European Contries») fecha de 2009, y por tanto no contempla la evolución de la situación en la década que ha seguido a la crisis financiera mundial. En este documento informan de que «la medida de trabajadoras sexuales migrantes a lo 
largo de Europa es aproximadamente del 47\% al 50\%» (TAMPEP, 2009), con claras disparidades entre orígenes - siete de cada diez proceden de regiones de Europea del este y central, y fundamentalmente Rumanía, Rusia y Bulgaria. En el caso de España, por otro lado,

el porcentaje de trabajadoras sexuales migrantes [...] se ha incrementado [...], ya que hoy en día el $90 \%$ de las trabajadoras sexuales son migrantes. Aunque las principales regiones de origen de las personas migrantes se han mantenido estables en los últimos años ( [...] Nigeria, América Latina y los países caribeños, estando Ecuador, Colombia y la República Dominacana especialmente presentes), [...] el segundo mayor grupo regional de trabajadoras sexuales migrantes (24\%) es de los nuevos países de la UE, principalmente Rumanía y Bulgaria (TAMPEP 2009: 26).

Por otro lado, Dolores Juliano (2004) señala la heterogeneidad de las redes (familiares, comerciales y coercitivas, que son las que se asimilan normalmente al concepto de «trata» más popularizado) a través de las cuales las personas migrantes acceden a los países europeos; también la investigación de López Riopedre (2016) da pistas sobre las «redes informales de apoyo» de las personas migrantes. Laura Agustín (2006) recuerda que «la industria sexual, mayormente desregularizada y ajena a la contabilidad del Estado, opera por definición a través de redes informales», siendo esta «la clave de la vulnerabilidad de las trabajadoras migrantes». Es por ello que los colectivos de trabajadoras sexuales y las trabajadoras sexuales que exigen sus derechos hacen hincapié en «escapar de las leyes punitivistas» y señalan «la criminalización del trabajo sexual como uno de los principales impulsores de la migración» (NSWP, 2018), así como «las detenciones arbitrarias amparadas por la Ley de Extranjería» y la violencia policial que alienta (Sánchez Perera, 2018) y los «intentos de limitar la migración, que producen obreros indocumentados que carecen de derechos laborales» (Mac y Smith, 2018). Estas autoras citan a las investigadoras Nassim Majidi y Saagarika Dadu-Brown, que afirman que las políticas fronterizas anti-inmigración crean «nuevas relaciones migrantes-contrabandistas», con el añadido que «los contrabandistas se van a aprovechar de un cierre de fronteras o de una restricción para subir los precios» (2017). Laura Oso Casas describe estas relaciones entre el trabajo sexual y el estatus irregular de las personas migrantes (en su caso, latinoamericanas) como «un laberinto circular» (2008). La vulnerabilidad que supone para las mujeres migrantes este estatus, y la violencia a la que las somete, es explicado por ellas con insistencia (Aranda et al., 2021).

En lo que sigue consideraremos el trabajo sexual en general.

\section{TRABAJO SEXUAL.}

\section{1. Mitificaciones feministas, malentendidos sociológicos}

Como decimos, la falta de datos fiables en torno a la realidad del trabajo sexual -en todas sus formas- se cruza aquí con la víscera social. Este cruce tiende a 
asumirse bajo la forma de dos posturas distintas y hasta contradictorias, conocidas como «regulacionista» y «abolicionista» (ver: Europa Press, 2019). Ambas parecen ser las únicas formas de entender la realidad social de la prostitución, hasta el punto de que toda aproximación parece ignorar el efecto real de la otra en el debate (Gimeno, 2012:45 y ss.).

Mac y Smith (2018:22-30) relatan la mitificación ideológica de la prostitución que han ofrecido a menudo las feministas «abolicionistas» de las mujeres prostitutas, deshumanizando a las mujeres y limitando su realidad a su cuerpo, haciendo una eterna reducción en cascada: todo trabajo sexual sería prostitución y toda prostitución sería forzada; en consecuencia, toda prostitución sería violación y explotación sexual. Hay versiones que añaden un último tramo a este esquema: el tráfico de mujeres [con fines de explotación sexual]. Resulta habitual que, en esta pendiente resbaladiza, se confundan, por la naturaleza del enfoque, la reducción («todo $x$ se explica por $y »)$ con la analogía («todo $x$ es en una forma de $y »)$. Algunos ejemplos incluyen (pero no se limitan) a Kate Millet (1976) o Sheyla Jeffreys (2011), y en el caso de España Ana de Miguel (2015:48 y ss.) o Rosa Cobo (2016:906). También en la búsqueda de trabajos en torno a la prostitución -sea sobre representación discursiva, realidad de las prostitutas, etc- se hace patente la confusión permanente entre la «prostitución»y la «trata».

Una consecuencia inmediata de esta confusión es la dificultad para plantear políticas que frenen o terminen con la trata y no ataquen a la vez a las mujeres que se prostituyen. (Veremos lo fundamental que resulta el concepto de «vulnerabilidad»). Por otra parte, una presuposición habitual aquí es que el trabajo sexual ha de ser, por su propia naturaleza, algo indeciblemente detestable, algo sencillamente inmoral y que nadie querría hacer. Esto no sólo debe ser puesto entre paréntesis en relación a las circunstancias, como veremos, sino que depende también de que las propias mujeres prostitutas no tengan voz. Un ejemplo: «para 2012, sólo en EEUU, el presupuesto total de las treinta y seis principales organizaciones contra el tráfico de mujeres era de 1.2 mil millones de dólares, al tiempo que el Gobierno Federal destina [a lo mismo] por año de 1.2 a 1.5 mil millones de dólares. La mayor parte de este dinero se gasta en campañas, en contraste al apoyo de las supervivientes: para 2014, Estados Unidos sólo disponía de mil camas en total para las víctimas de tráfico» (Mac y Smith, op.cit.:58-59).

Este exceso es acusado por Mac y Smith (op.cit.:59-61) y por Agustín (2002; 2009), que recuerdan que la victimización e infantilización deshumanizadoras -dos representaciones por las que se niega agencia y voz- de las mujeres prostitutas sirven para alimentar los tópicos y la confusión entre la prostitución y el tráfico, haciendo de las políticas en torno a la primera una «operación de rescate» que no redefine la prostitución en términos estructurales, sino que de hecho -y en la medida en que sólo se hace hincapié en esas mujeres-, la mantiene. Las primeras (op.cit.:74-83) señalan igualmente el origen racista propio de parte de las campañas y las políticas «abolicionistas». Añaden: «la población LGTBQ y negra, [y] las prostitutas por voluntad tienden a dejarse fuera de la categoría de inocente y, en consecuencia, la violencia hacia estos grupos [dentro de la prostitución] parece menos dañina» (íb.:60). 
Sin embargo, desde otros supuestos más cercanos al «regulacionismo» se suele defender la «libertad» abstracta y acrítica, no problematizada, de las propias personas que se dedican a la prostitución. Agustín (2002:115) dice que «además de los factores económicos que pueden impulsar a esos migrantes, existe el deseo de conocer el mundo.. Ningún tipo de determinismo explica por completo el fenómeno humano de la elección». Pero esto obvia por completo las restricciones estructurales que hemos estudiado, y las reduce a un simple «campo de batalla», como si los propios agentes sociales no contaran con ellas realmente (y a pesar de que el texto está dedicado a contar cómo esto sí sucede). López Riopedre (2016) afirma que «esta realidad [los «deseos subjetivos» de «viajar»] aparece constantemente en las trayectorias y narraciones de las migrantes, por lo que pretender reducir sus proyectos migratorias a las... constricciones estructurales equivale a deshumanizar a estas mismas migrantes». Y aunque esta crítica a las interpretaciones de la «sobredeterminación estructural» es válida, la argumentación del autor sigue girando en torno a la misma idea problemática de la estructura como tabula rasa.

Al comienzo del artículo, Agustín (2002:110) relata -sin dar especificidades de ninguna clase- las diferentes formas en las que las redes de prostitución se imbrican, haciendo hincapié en la diversidad y complejidad que les es propia, y afirma: «en una industria que se caracteriza por sus ambigüedades, es conveniente no perpetuar el supuesto clásico de mujer-sexoservidora/ hombre-cliente». Y aunque existe la prostitución masculina, esta es minoritaria (Ranea, 2012), y además se ha asociado históricamente a la homosexualidad masculina y las mujeres trans (Kaye, 2006). En todo caso, la única encuesta por la que poseemos algún dato al respecto (CIS, 2009:21) señala que un tercio de los hombres de la muestra habría pagado por sexo al menos una vez, mientras que sólo una porción marginal de mujeres respondió lo mismo. Ahora bien, las respuestas siguientes en torno al abuso sexual sugieren lo que por otro lado es lógico: la población femenina no responde de forma muy distinta de la masculina, ya sea esto por el estigma social de la sexualidad femenina, por falta de un marco cognitivo que sea favorable a esta interpretación, o por ambas. (Hay que destacar que, si bien es cierto que existe una prostitución masculina, esta no forma una muestra destacable, siendo en cualquier caso inmigrantes en situación irregular quienes -también debido a las dificultades asociadas a su situación «libre»- se han estudiado; ver F. Triángulo, 2006).

Así, aunque la perspectiva del «trabajo sexual» funciona adecuadamente para pensar la complejidad de este conjunto diverso de fenómenos, suele usarse de forma acrítica, de manera que acompaña a diferentes malentendidos sociológicos. Al margen de las (a menudo) graves mitificaciones que hemos mencionado, la crítica «abolicionista» ha proporcionado algunos de los aciertos más fundamentales en lo que hace a la lógica social que opera en la prostitución.

\section{2. Trabajo, trabajo sexual y cuerpo}

Quizá el problema fundamental a la hora de plantear si la prostitución es o no efectivamente un trabajo -reflexión en la que no nos hemos detenido- es la 
propia naturaleza del «trabajo» como tal. En una sociedad capitalista -en la que la mercancía supone el objeto nuclear, y el capital deviene en sujeto automático-, el trabajo cumple la función de valorizar la mercancía, se materializa en ella y reside, en potencia, en el cuerpo humano (Marx, 2008). El carácter de «emancipación» del capitalismo, su destrucción de los vínculos de dependencia consiste justo en que, incidentalmente, la propiedad que da valor al trabajador es ahora su fuerza de trabajo, y no la totalidad corporal, en carne y servidumbre. Esto cambió con la «terciarización» y la «financiarización» de la economía, siendo estas sin embargo partes específicas del entramado económico-social (Jappé, 2016) y legal (Cukier, 2019). El trabajo sexual se incorpora a este esquema a través de la actividad sexual en su heterogeneidad como «servicio».

Desde el punto de vista feminista, el cuerpo cuya actividad se vende es específicamente generizado, y está situado en términos de clase y de raza. El «cliente» es un hombre casi siempre, mientras que la «trabajadora sexual» es una mujer en la mayoría de los casos. Mac y Smith recuerdan, como trabajadoras sexuales, que

grupos marginados, como la comunidad LGTB, están sobre-representados en el trabajo sexual. La discriminación, el rechazo y los abusos, en el hogar y otros contextos más amplios, empeoran la precariedad y vulnerabilidad que sufren en una sociedad homófoba y tránsfoba, dejando la prostitución como una de las pocas salidas posibles. En particular, las mujeres trans suelen descubrir que el empleo formal está fuera de su alcance (op. cit., 50).

El único estudio sobre la población trans que reseña -con la limitación propia del contexto de la investigación- de forma tangencial la prevalencia de la prostitución entre esta comunidad (Guzmán-Parra et al., 2016) marca una relación entre esta condición sexual y el género (mujeres; hombres) con la prostitución, siendo la transfeminidad un factor de riesgo. Sucede lo mismo con la raza, determinada por la procedencia, como hemos visto antes. (Ver también: Brooks et al., 2006; Ryan, 2006).

Esto muestra la complejidad de la realidad de la prostitución, pero también señala ciertos puntos clave: (a) la disposición masculina a pagar por sexo y (b) la vulnerabilidad económica y social, (c) basada en el género, (d) condición sexual y (e) procedencia, de quien vende sexo. A falta de un estudio que enfoque desde distintas metodologías un fenómeno tan subterráneo, estigmatizado y complejo, estos parecen ser los factores que inciden en la prostitución.

Esto supone que los cuerpos de las mujeres, y su expresión de feminidad, son parte de un pacto general determinado por los hombres: el contrato sexual. Carol Pateman (2019) estudia, en una alusión irónica y despectiva al texto de Rousseau, el contrato sexual que organiza a las mujeres socialmente en relación a los hombres. De acuerdo con la autora,

el pacto originario es tanto un pacto sexual como un contrato social; es sexual en el sentido de que es patriarcal [...]: el contrato establece el derecho político de los varones sobre las mujeres. También es sexual en el sentido de que establece un orden de acceso de los varones al cuerpo de las mujeres. El contrato original crea 
lo que denominaré [...] la «ley del derecho sexual masculino». El contrato [...], lejos de oponerse al patriarcado, es el medio a través del cual se instituye el patriarcado moderno. (2019:37)

Esta perspectiva marca las coordenadas a partir de las cuales debe leerse el fenómeno de la prostitución. El lugar de las esposas pasa fácilmente al de las prostitutas, bajo este contrato sexual; «la prostitución es parte integral del capitalismo patriarcal» (íb.:347). En este sentido, «la historia del contrato sexual revela que la construcción patriarcal entre masculinidad y fe-minidad es la diferencia política entre libertad y sujeción, y que el dominio sexual es el medio más importante por el que los varones afirman su virilidad» (íb.: 378-379). La fortaleza de la crítica de Pateman reside, sin embargo, en el nivel de abstracción en que se mueve: de nuevo, no es capaz de concretizar la realidad y la heterogeneidad de las mujeres de las que habla. A pesar de ello, no cae en el problema criticado de equiparar distintas clases de trabajo sexual; y es capaz de hablar de masculinidad y feminidad sin partir de supuestos esencialistas.

Doezema (2010) afirma sobre esa retórica que «el eco [...] de la pornografía es notable. La prostituta no sólo tiene carencias [...]; ella es carencia». El efecto sociológico de este discurso es claro, en la medida en que el cuerpo femenino es un cruce siempre marcado por la sexualidad y el deseo masculinos. Por supuesto, la dialéctica entre los géneros presupone y reproduce una oposición estable; la prostitución es una consecuencia natural de la disposición sistémica de los hombres a acceder no a los cuerpos femeninos como tales, sino a las representaciones encarnadas de su condición femenina. Si bien esta afirmación puede parecer atrevida desde la ingenuidad sociológica, hay que enfatizar que la prostitución se basa en (y pone en juego) los significados que posee y adquiere la subjetivación masculina en su relación con los cuerpos feminizados (Ranea, 2012).

Gómez-Suárez, Pérez-Freire y Verdugo-Matés (2016) establecen cuatro tipos básicos de hombres que pagan por sexo: (a) el misógino, hombre de derechas con nivel educativo bajo-medio y salario bajo; (b) el consumidor, hombre joven con un discurso anti-sexista y liberal, de centro-izquierdas, que se plantea la prostitución como una simple transacción; (c) el amigo, hombre joven de izquierdas, que es «[capaz] de humanizar a las mujeres en prostitución y de «empatizar» con ellas» y su situación sin alternativa, y que «recrean los vínculos de «ligoteo casual»»; (d) el crítico, hombre de izquierdas de alrededor de 30 años, ex putero que reconoce las desigualdades implícitas en la prostitución y se arrepiente de haber pagado por sexo. Aquí se puede ver la relación entre masculinidad y feminidad a través del cuerpo de la prostituta y por medio del contrato sexual implícito.

La vigilancia y la sujeción del cuerpo de las trabajadoras sexuales es uno de los mecanismos fundamentales para el mantenimiento del contrato sexual que mencionamos. Para que aquel sea atado a los imperativos de la masculinidad -que es la base de su realidad social, incluso en los casos de prostitución masculina a los que hemos hecho referencia-, debe seguir viéndose como una excrecencia más de ella, como algo «pre-político..., una realidad que transita entre lo natural y lo social» (Cobo, 2016:900). Parte de este relato, que define a las propias mujeres prostitutas 
-y debe ser redefinido por ellas mismas-, es el de la significación de los espacios en relación a la significación social de la prostitución (ver: García, 2017).

En este sentido, resulta notable el caso de Murcia, que vemos a continuación.

\section{3. Murcia: las consecuencias empíricas de la prohibición}

El 8 de octubre de 2013, una Comisión del Ayuntamiento de Murcia aprobó la Ordenanza para luchar contra la prostitución en el municipio. De acuerdo con la exposición de motivos, sus objetivos son dos: "preservar los espacios públicos como lugares de convivencia, civismo e igualdad», y con ello «evitar actividades de explotación sexual que difunden una imagen... de la mujer como mero objeto sexual». Esto último resulta particularmente interesante porque se basa en tres Leyes específicas: la LO 1/2004 de Medidas de Protección Integral contra la Violencia de Género, la LO 3/2007 para la igualdad efectiva de hombres y mujeres y la Ley 7/2007 para la Igualdad entre Mujeres y Hombres, y de Protección contra la Violencia de Género en la Región de Murcia.

Lo primero que llama la atención, por la inmediatez, es la individualización de las causas de la prostitución, que además hacen de las propias mujeres prostitutas participantes activas en su propia «explotación sexual». Esta estrategia conservadora contradice toda la evidencia citada, que sitúa a las mujeres, y especialmente las mujeres migrantes, como una población de riesgos específicos. La vulnerabilidad urbana (Alguacil y Camacho, 203), como un marco que integra los factores de vulnerabilidad social dentro del espacio urbano, organiza a mujeres y a hombres de manera distinta, en relación a la feminización del trabajo y al trabajo de cuidados al que hemos hecho referencia en secciones anteriores, y de ahí la disparidad por género.

Por otro lado, el par «convivencia/ civismo» se usa aquí específicamente para mantener la prostitución fuera del espacio público, lejos de la mirada social. No se menciona en ningún momento qué causa, en términos generales, el fenómeno de la prostitución, con que se asume que constituye un fenómeno natural, que sólo debe restringirse al ámbito privado. No se ataca la realidad concreta, sino la institución, en cuanto se relaciona con determinado capital social.

El Colectivo de Apoyo a las Trabajadoras Sexuales (CATS, 2014) redactó más adelante un informe para evaluar el primer año de aplicación de la Ordenanza en la ciudad. De acuerdo con la asociación, «la principal razón para que el Ayuntamiento de Murcia haya acometido la regulación del ejercicio de la prostitución de calle en todo el municipio han sido las demandas vecinales de uno de sus barrios históricos: El Carmen». Este barrio histórico -con la edifi-cación del ferrocarril en el siglo XIX y la industrialización en el siglo XX-vio empeorar sus condiciones económicas y de vivienda tras la ampliación de las zonas del norte. Así, cuando comenzó el período migratorio de los noventa -con el asentamiento de poblaciones de origen heterogéneo-, El Carmen acogió a buena parte de estas comunidades. En general, este es un barrio cuya población, tanto autóctona como migrante, concentra una enorme "vulnerabilidad sociodemográfica». Estas condiciones fomentan circuitos de «economía informal, como es el ejercicio de la prostitución» (íb.:10). 
Las mujeres afectadas estudiadas por el trabajo son 44, de las cuales 31 son marroquíes, 6 son guineanas, 3 son rumanas, y las 4 restantes de otras nacionalidades. Más de la mitad de ellas tienen entre 35 y 44 años; un cuarto de ellas, todas marroquíes, reconocen ser víctima de violencia de género; además, más de la mitad tienen cargas familiares (íb.:34-35). El informe destaca que todas estas mujeres se prostituyen sin la coacción de terceros, y que la mayoría «quiere dejar la prostitución y buscar otras alternativas en el mercado de trabajo regularizado en el que se reconozcan sus derechos laborales» (íb..24-25).

Las multas de la Ordenanza han tenido unas consecuencias muy claras:

Buena parte [de estas mujeres] han trasladado su área de trabajo y captación de clientes a otras zonas más «toleradas». Estos lugares suelen estar más alejados del núcleo urbano y por tanto, son zonas más desprotegidas en las que aumenta el riesgo para las personas que ejercen la prostitución ya que hacen más precarias y peligrosas las condiciones de desempeño de su actividad (íb.)

Y esto porque la Ordenanza ni siquiera discrimina las situaciones de prostitución de otras situaciones: hay mujeres que han sido multadas por «entrar o salir de sus domicilios» o bien por «ser perseguidas por un cliente por la calle», además de por ir acompañada de familiares o por huir de la propia policía (íb.: 39-41). Al estar pensada para expulsar a las mujeres pobres de su zona y por tanto ignorar sus circunstancias, la Ordenanza empeora más sus condiciones. Es un ejemplo directo del rol de las políticas que prohíben el trabajo sexual, que en ninguno de los casos funciona mejorar las condiciones de las mujeres trabajadoras.

\section{CONCLUSIONES PARA PENSAR EL TRABAJO SEXUAL.}

La globalización, al redefinir la relación entre localidad y mundo y del territorio en su propia composición, resignifica el rol y la agencia de las personas en relación a sus estructuras. En este marco, los factores sociales suponen una realidad más compleja, que se extiende a través de nuevos vínculos de dependencia. Al mismo tiempo, la privatización neoliberal y las nuevas formas de contratación han limitado enormemente las posibilidades de la clase trabajadora. En esta composición compleja, la prostitución juega un papel importante a la hora de marcar las relaciones entre géneros -en base a la legitimidad sexual masculina y a la subordinación de las mujeres, en situación de vulnerabilidad-, y especialmente en relación a la migración. Esto parece especialmente claro en Murcia, como ciudad del sur dentro de la Europa del Sur.

Las dificultades en el estudio de este trabajo son muchas, comenzando por el estigma que sufre la prostitución como institución, y especialmente las mujeres prostitutas. Es necesario plantear, desde la seriedad y el estudio riguroso, políticas que mejoren las condiciones socio-económicas de la población, e incidir en los factores que ocasionan la vulnerabilidad -como son el género y la condición de migrante, especialmente en su intersección-; pero para ello es necesario que comprendamos la díficil y heterogénea realidad que tratamos de abordar. Esto no puede hacerse 
exclusivamente desde las entrañas, sino que debe organizarse un abordaje comprensivo, relacional:

Esta constatación [la del contrato sexual] no puede llevarnos a ver a las prostitutas como las que "colaboran y refuerzan el patriarcado», ni como «las víctimas por excelencia de él», como plantean ciertas feministas. Su visión considera a las prostitutas seres pasivos, meras receptoras de la ideología patriarcal. Estas consideraciones olvidan que todas, de una $\mathrm{u}$ otra forma, vivimos situaciones de subordinación que intentamos combatir como podemos. [...] Nuestra existencia es un extraño equilibrio entre la rebelión y el pacto, y no por ello se puede decir que «colaboremos con el patriarcado» (Garaizabal, 2007).

Un debate del calado y alcance necesarios para pensar en y transformar las condiciones que estructuran las vidas de todos los agentes implicados en la institución del trabajo sexual sólo puede plantearse, análogamente, desde una perspectiva integral. O, concretando más esta propuesta, exige desafiar los prejuicios para analizar científicamente los diversos elementos que componen este entramado y que une la globalización y la migración con el género y la construcción de imaginarios asociados a feminidad y masculinidad -no sólo en el sentido de «aquello que se atribuye a mujeres y hombres en tanto que tales» sino más especialmente en el sentido de «aquello que constituye el hecho mismo de ser un sexo-género»-, siempre limitados por su estructuración pero abiertos a la reificación, y siempre relacionados con el estatus de clase y la posición ocupada en términos sociales.

También merece un comentario el uso que se ha hecho del término «trabajo sexual» a lo largo de este estudio, ya que es un elemento central a la hora de establecer este debate y darle un enfoque complejo. El concepto de «trabajo sexual» se ha empleado tradicionalmente como un acto político que reivindica la legitimidad de la actividad sexual considerada como trabajo. Acuñado por Carol Leigh en un libro coral y popularizado al momento, Frédérique Delacoste, coeditora, afirmaba que este término "pretendía crear un espacio en el que «la prostitución» no fuera entendida de forma automática como una metáfora de la auto-explotación» (1987); para la propia Carol Leigh, se trataba de «crear una atmósfera de tolerancia, dentro y fuera del movimiento de las mujeres, para las mujeres que trabajan en la industria sexual» (en Nagle, 1997). El objetivo era, por tanto, enfrentar el estigma propio de las trabajadoras sexuales.Sin embargo, aquí se trata de evitar este uso, pretendiendo cubrir cuatro objetivos fundamentales: (i) no deshumanizar a las trabajadoras sexuales empleando eufemismos; (ii) desplazar en el proceso los mitos asociados popularmente a la prostitución y los prejuicios relacionados a las trabajadoras sexuales en general; (iii) englobar distintas formas de actividad relacionadas con la sexualidad, que deben ser comprendidas en su heterogeneidad y con una sensibilidad particular para (iv) permitir entender mejor el trabajo sexual como institución y fenómeno, situando la perspectiva en las condiciones que lo construyen y sostienen, y permitiendo un enfoque amplio y abierto a la complejidad interna. Es posible respetar la contribución directa de las trabajadoras sexuales e incorporarla al estudio. 
Hay que señalar, para defender este argumento de las críticas habituales, que el concepto de «trabajo sexual» no es problemático en ningún sentido mientras se emplee con un enfoque comprensivo como el que se trata de aplicar aquí. Nada en este uso exige vaciar el concepto de "consentimiento», sino más bien complejizarlo, e incluso problematizar las perspectivas más simplistas, ya que lo sitúa en sus condiciones estructurales y permite analizarlas en su debido contexto. Nada en el planteamiento de que «el trabajo sexual es trabajo» implica que tenga que ser «digno», siquiera aceptable; al contrario, permite dar sentido al contexto en el cual se negocia el «servicio» y recuerda que todo trabajo, en una sociedad de clases, es un trabajo apropiado por un otro con la capacidad de apropiarse de él; permite comprender que, bajo condiciones sociales adecuadas, la sexualidad no es apropiable en este sentido. Este uso tiene la virtud de ser útil a las perspectivas marxista y feminista, de modos que no han sido todavía explorados adecuadamente.

Por último, dado que la práctica científica está siempre unida a unos intereses implícitos de investigación, un proyecto de construcción de tal conocimiento científico ha de ser siempre consciente de tales intereses y ha de estar guiado por ellos. En la misma medida en que todo conocimiento científico ha de ser situado, los principios de comprensión y de transformación de las estructuras sociales han de ser supuestos fundamentales para la práctica científica, que nunca es -pero mucho menos en las ciencias sociales- una práctica social aislada. Desde la perspectiva de una teoría social crítica, el análisis de un fenómeno social -en este caso, las instituciones del trabajo sexual en general y de la prostitución en particular- es siempre una consecuencia de una injusticia social que ha de ser conceptualizada y revolucionada. Tal y como asumía Marx (1968), «esta crítica no se comporta como un fin en sí, sino simplemente como un medio. Su pathos es la indignación; su tarea, la denuncia».

\section{BIBLIOGRAFÍA Y DOCUMENTACIÓN}

Agustín, Giorgio (2016). Walter Benjamin y el capitalismo como religión. Disponible en culturamas.es: https://www.culturamas.es/blog/2016/10/16/giorgio-agamben-walter-benjamin-y-el-capitalismo-como-religion/ (Fecha de consulta: 18 de mayo de 2020)

Agustín, Laura (2002). «La industria del sexo, los migrantes y la familia europea», en Guasch, Oóscar y Viñales, Olga (2002), Sexualidades: Diversidad y Control Social, Barcelona: Bellaterra.

Agustín, Laura (2006). «Migration and Mobility», en Ditmore, Melissa (ed.) (2006), Encyclopedia of Prostitution and Sex Work, Greenwood., pp. 308-314.

Agustín, Laura (2009). «The conundrum of women's agency: migration and the sex industry», en CAmpbell, Rosie y O'Neill, Maggie (eds.) (2009). Sex Work Now, Cullompton: Willan.

Alguacil, Julio, y CAMACHO, Javier (enero-abril 2014). «La vulnerabilidad urbana en España. Identi€cación y evolución de los barrios vulnerables», en EMPIRIA. Revista de Metodología de Ciencias Sociales, n. 27, pp. 73-94. 
Aranda López, María, Moreno Molina, David, Fernández Contreras, Teres y Morcillo Martínez, Juana $M^{\mathrm{a}}$ (2021). «Sexual and gender-based violence in migration context: Needs, gaps and good practices», en Revista Internacional de Apoyo a la Inclusión, Logopedia, Sociedad y Multiculturalidad, v. 7, n. 1, enero 2021.

BARAÑANo, Margarita, y MARCHetTI, Sabrina (2016), "Perspectivas sobre género, migraciones transnacionales y trabajo: rearticulaciones del trabajo de reproducción social y de cuidados en la Europa del Sur», en Investigaciones Feministas, v. 7, n. 1, pp. 9-33. Disponible en: https://iris.unive.it/retrieve/ handle/10278/3677790/78075/53094-99880-3-PB.pdf (fecha de consulta: 20 de mayo de 2020).

Briz, Mamen, y GARAIzÁbAL, Cristina (2007), La prostitución a debate, Madrid: Talasa.

Brooks, Siobhan, Ditome, Melissa, y Koken, Juline (2006). «Race and Ethnicity», en Ditmore, Melissa (ed.) (2006), Encyclopedia of Prostitution and Sex Work, Greenwood., pp. 377-382.

Camacho, José, Rodríguez, Mercedes, y Hernández, Manuel (2018). «El sistema de atención a la dependencia en España: evaluación y comparación con otros países europeos», en Cuadernos Geográficos, n. 42, pp. 37-52. Disponible en: https:/ / www.ugr.es/ cuadgeo/docs/articulos/042/042-002.pdf (fecha de consulta: 17 de mayo de 2020).

Campbell, Rosie y O'NeIll, Maggie (eds.) (2009). Sex Work Now, Cullompton: Willan.

CATS (2014). Informe de evaluación de la «Ordenanza...». Disponible en http:/ /www. asociacioncats.es / wp-content/uploads/2018/04/EVALUACI\%C3\%93N-DELA-ORDENANZA-CONTRA-LA-PROSTITUCI\%C3\%93N-EN-EL-MUNICIPIO-DE-MURCIA.pdf (fecha de consulta: 21 de mayo de 2020).

CIS, Estudio $n^{\circ}$ 2.78: Encuesta Nacional de salud sexual (Enero 2009).

Сово, Rosa (2016). «Un ensayo sociológico sobre la prostitución», en Política y Sociedad, v. 53, n. 3, pp. 897-914.

CUKIER, Alexis (28 de febrero de 2019). «Explotación, marxismo y derecho laboral», documento disponible en Perspectiva. Blog de Investigación Filosófica: https:/ / bperspectiva.wordpress.com/2019/02/28/explotacion-marxismo-y-derecholaboral-iii-por-alexis-cukier/ (fecha de consulta: 21 de mayo de 2020).

De Miguel, Ana (2015). Neoliberalismo sexual, Madrid: Cátedra.

Dellacoste, Frédérique y Alexander, Priscilla (ed.) (1998), Sex work: writings by women in the sex industry, San Francisco: Cleis Press.

Ditmore, Melissa (ed.) (2006), Encyclopedia of Prostitution and Sex Work, Greenwood.

Doezema, Jo (2006). «Abolitionism», en Ditmore, Melissa (ed.) (2006), Encyclopedia of Prostitution and Sex Work, Greenwood., pp. 4-7.

Doezema, Jo (2010), Sex Slaves and Discourse Masters: The Construction of Trafficking, New York: Zed Books.

Dussel, Enrique (2013). 16 tesis de Economía Política, Buenos Aires: Docencia.

Europa Press (2019): «El debate sobre la prostitución llega al Congreso: ¿Abolición o regulación?», documento disponible en: https://www.europapress.es/ep- 
social/igualdad/noticia-debate-prostitucion-llega-congreso-abolicion-regulacion-20190217130237.html (fecha de consulta: 20 de mayo de 2020).

Fundación Triángulo, Trabajadores Masculinos del Sexo: Aproximación a la prostitución masculina en Madrid (2006), documento disponible en: http:/ / www.fundaciontriangulo.org/documentacion/documentos/trabajadoresmasculinos.pdf (fecha de consulta: 25 de mayo de 2020).

GARAIZÁBAL, Cristina (2007). «El estigma de la prostitución», en BRIZ, Mamen, y GARAIZÁbAL, Cristina (eds.) (2007). La prostitución a debate, Madrid: Talasa, pp. 43-55.

GARcía, Leticia (2017). "Territorios de la prostitución: de problemas a contenidos», en Geografía. XVI Encuentro de Geógrafos de América Latina, 26 al 29 de abril, UMSA: La Paz.

Gimeno, Beatriz (2012), La prostitución, Barcelona: Bellaterra.

Global Network of Sex Work Projects, Research for Sex Work, n. 14 (2015). Documento disponible en: https:/ / www.nswp.org/sites/nswp.org/files/R4SW\%20 2015_issue14_PDFV.pdf (fecha de consulta: 19 de mayo de 2020).

Gómez-SuÁrez, Águeda, Pérez-Freire, Silvia y Verdugo-Matés, Rosa María (mayo-agosto 2016), «Dominación, sexualidad masculina y prostitución en España: ¿por qué los hombres españoles consumen sexo de pago?», en Convergencia: Revista de ciencias sociales, n. 71, pp. 149-174.

Guasch, Oóscar y Viñales, Olga (2002), Sexualidades: Diversidad y Control Social, Barcelona: Bellaterra.

GuZMÁn-PARRA et al (abril 2016), «Sociodemographic Characteristics and Psychological Adjustment Among Transsexuals in Spain», en Archives of Sexual Behavior, v. 45, Issue 3, pp 587-596.

Instituto Nacional de Estadística (2008), Documentos de trabajo 2/08. Informe Encuesta Nacional de Inmigrantes (ENI - 2007), Madrid. Documento disponible en: http:/ / www.ine.es/daco/daco42/inmigrantes/informe/eni07_informe.pdf (fecha de consulta: 24 de mayo de 2020).

Herrera, Gioconda y Ramírez, Jacques (ed.) (2008), América Latina migrante: Estado, familias, identidades, Quito: Ministerio de Cultura del Ecuador.

Hochschild, Arlie Russell (2001). «Las cadenas mundiales de afecto y de asistencia y la plusvalía emocional», en Hutton, Will y Giddens, Anthony. (2001), En el límite: la vida en el capitalismo global, Barcelona: Tusquets.

JAPpÉ, Anselm (2016). Las aventuras de la mercancía, Logroño: Pepitas de calabaza.

JEFFREYs, Sheyla (2011), La industria de la vagina. La economía política de la comercialización global del sexo, Buenos Aires: Paidós.

Juliano, Dolores (2004). «El peso de la discriminación: debates teóricos y fundamentaciones», en Osborne (ed.) (2004), Trabajador@s del sexo. Derechos, migraciones y tráfico en el siglo XXI, Barcelona: Bellaterra.

Kaye, Kerwin (2006). «Male Prostitution», en Ditmore, Melissa (ed.) (2006), Encyclopedia of Prostitution and Sex Work, Greenwood., pp.275-281.

López, Isidro, y Rodríguez, Emmanuel (2010), Fin de ciclo. Financiarización, territorio y sociedad de propietarios en la onda larga del capitalismo hispano (1959-2010), Madrid: Traficantes de Sueños. 
López Riopedre, José López (diciembre 2016). «Trabajo sexual transnacional: consecuencias de las políticas criminalizadoras de la prostitución y de la crisis económica española sobre las trabajadoras sexuales migrantes», en Revista electrónica del Departamento de Derecho de la Universidad de La Rioja (REDUR), n. 14, pp. 67-86.

Mac, Juno, y Smith, Molly (2018), Revolting Prostitutes. The Fight for Sex Workers' Rights, London: Verso.

MajIdI, Nassim y DAdu-Brown, Saagarika (2017). «Human smugglers roundtable: On border restrictions and movement». Documento disponible en: https:/ / www.opendemocracy.net/en/beyond-trafficking-and-slavery/select-answersquestion-two/ (fecha de consulta: 4 de marzo de 2021).

MARx, Karl (1968). Introducción a la crítica de la filosofía del derecho de Hegel, Buenos Aires: Claridad.

Marx, Karl (2008), El capital: Crítica de la economía política (Libro I, 2 v.), México: Siglo XXI.

Millán Vázquez de la torre, Millán, Del Pilar Santos, Manuela y Pérez NaRANJO, Leonor María (abril/junio 2015). «Análisis del mercado laboral femenino en España: evolución y factores socioeconómicos determinantes del empleo», en Papeles de Población, vol. 21, n. 84. Documento disponible en: http://www. scielo.org.mx/scielo.php?script=sci_arttext\&pid=S1405-74252015000200008 (fecha de consulta: 2 de junio de 2020).

Millet, Kate (1976), The Prostitution Papers: A Quartet for Female Voice, New York: Ballantine Books.

Molpeceres Álvarez, Laura (2012), «Situación laboral de las mujeres inmigrantes en España», en Cuadernos de Relaciones Laborales, vol. 30, núm. 1, pp. 91-113.

NAGLE, Jill (ed.) (1997), Whores and other feminists, Routledge: New York.

NSWP (Global Network of Sex Work Projects) (2018). «Briefing Paper: Migrant Sex Workers». Documento disponible en: https:/ /www.nswp.org/sites/nswp. org/files/migrant_sex_workers_bp_spanish_final.pdf (fecha de consulta: 4 de marzo de 2021).

Orozco, Amaia (2007). Cadenas globales de cuidado (Documentos de trabajo 2), Rep. Dominicana: Instituto Internacional de Investigaciones y Capacitación de las Naciones Unidas. Documento disponible en: http://www.mueveteporlaigualdad.org/publicaciones/cadenasglobalesdecuidado_orozco.pdf (fecha de consulta: 2 de junio de 2020).

Osborne, Raquel (2004), Trabajador@s del sexo. Derechos, migraciones y tráfico en el siglo XXI, Barcelona: Bellaterra.

Oso, Laura (2008). «Mujeres latinoamericanas en España y trabajo sexual: un laberinto circular», en Herrera, Gioconda y Ramírez, Jacques (ed.) (2008), América Latina migrante: Estado, familias, identidades, Quito: Ministerio de Cultura del Ecuador.

Oso, Laura, y PARella, Sonia (2012). «Inmigración, género y mercado de trabajo: una panorámica de la investigación sobre la inserción laboral de las mujeres inmigrantes en España», en Cuadernos de Relaciones Laborales, v. 30, n. 1, pp. 11-44. 
Pateman, Carol (2019), El contrato sexual, Madrid: Ménades.

PiketTy, Thomas (2014). El capital en el siglo XXI, Madrid: Fondo de Cultura Económica.

RANEA, Beatriz (2012), «La demanda en disputa. La construcción social de la masculinidad heterosexual y la prostitución femenina», en VÁzquez BeRMúdez, Isabel (coord.) (2012). Investigación y género, inseparables en el presente y en el futuro: IV Congreso Universitario Nacional Investigación y Género, Sevilla, 21 y 22 de junio de 2012 , pp. 1569-1586.

Ryan, Joelle Ruby (2006). «Transgender Sex Workers», en Ditmore, Melissa (ed.) (2006), Encyclopedia of Prostitution and Sex Work, Greenwood., 499-506.

SÁnchez Perera, Paula (2018). «El feminismo regulacionista no existe, pero el proderechos sí». Documento disponible en: https://www.eldiario.es/opinion/tribuna-abierta/feminismo-regulacionista-existe-pro-derechos_129_2212408.html (fecha de consulta: 4 de marzo de 2021).

SAssen, Saskia (2003). Contrageografías de la globalización, Madrid: Traficantes de Sueños.

SAssen, Saskia (2015). Expulsiones, Madrid: Katz.

Scoтt, Brett (2019). «La gentrificación del pago: la extensión de la red financiera digital», artículo en El Salto Diario. Publicado el 17/05/2019 en: https://www. elsaltodiario.com/dinero/gentrificacion-pago-extension-red-financiera-digital (fecha de consulta: 2 de junio de 2020).

TAMPEP (The European Network for the Promotion of Rights and Health among Migrant Sex Workers) (2009). "Sex Work in Europe: A Mapping of the prostitution scene in 25 European contries». Documento disponible en: https:/ tampep.eu/wp-content/uploads/2017/11/TAMPEP-2009-EuropeanMapping-Report.pdf (fecha de consulta: 4 de abril de 2021).

THEOdORE, Nik; PeCK, Jamie; BrenNer, Neil (marzo 2009), «Urbanismo neoliberal: la ciudad y el imperio de los mercados», en Temas sociales, v. 66. Documento disponible en http:/ / www.sitiosur.cl/r.php?id=898 (fecha de consulta: 2 de junio de 2020).

Valero-Matas, Jesús Alberto, Coca, Juan. R. y Valero-Oteo, Irene (abr.jun. 2014). «Análisis de la inmigración en España y la crisis económica», en Papeles de Población, v. 20 n. 80. Documento disponibe en: http://www.scielo.org.mx/ scielo.php?script=sci_arttext\&pid=S1405-74252014000200002 (fecha de consulta: 3 de junio de 2020).

\section{LEGISLACIÓN}

Ley Orgánica 1/2004, de Medidas de Protección Integral contra la Violencia de Género.

LEY ORGÁNICA 3/2007, para la igualdad efectiva de hombres y mujeres.

LEY 7/2007, para la Igualdad entre Mujeres y Hombres, y de Protección contra la Violencia de Género en la Región de Murcia. 
ORdenANZA PARA LUCHAR CONTRA LA PROSTITUCión EN EL MUNICIPIO DE MURCIA (8 de octubre de 2013). Documento disponible en: https:/ / www.murcia.es / documents/11263/242162/ORD_PROSTITUCION.pdf (fecha de consulta: 2 de junio de 2020).

Recibido el 23 de junio de 2020 Aceptado el 25 de marzo de 2021 BIBLID [1132-8231 (2021): 105-124] 